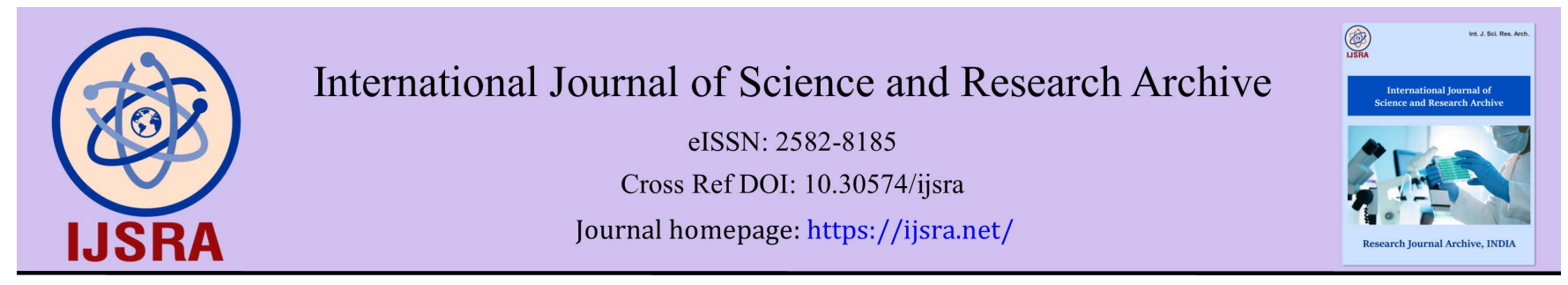

(REVIEW ARTICLE)

\title{
A systematic review of the variants of Sars-Cov-2
}

\author{
Mohammed Farhan Qureshi ${ }^{1, *}$ and Shoeb Qureshi ${ }^{2}$ \\ ${ }^{1}$ Prince Sultan Military Medical City, Division of Neonatology, Department of Pediatrics, Riyadh, Saudi Arabia. \\ ${ }^{2}$ Department of Research, King Saud Bin Abdulaziz University for Health Sciences, National Guards, Saudi Arabia.
}

International Journal of Science and Research Archive, 2022, 05(02), 009-017

Publication history: Received on 17 January 2022; revised on 24 February 2022; accepted on 26 February 2022

Article DOI: https://doi.org/10.30574/ijsra.2022.5.2.0050

\begin{abstract}
Before the end of December 2019, the epidemic was mainly localized in Wuhan, Hubei Province, China. Most of the cases in this stage were related to exposure to Huanan seafood market. "The virus is transmitted through human-to-human contact and rapidly spread across the world, and soon turned into a pandemic." Its symptoms can be divided to two main groups of majors (fever, cough, and dyspnea) and minors (anosmia, dysgeusia, headache, gastrointestinal symptoms, and skin lesions). Ever since, it has caused worldwide social and economic disruption, there are no universally recognized clinically useful antiviral drugs against COVID-19 so far (actually remdesivir showed good efficacy in many trials, but it needs to be further realized). Although there are several effective vaccines to prevent infection with SARS-CoV-2, efforts to develop medications and vaccines are continuing. Recently, a novel coronavirus, severe acute respiratory syndrome SARS-CoV-2, causing coronavirus disease 2019 (COVID-19), emerged in late 2019. During February 1-July 13, 2020, were identified 4,080 transmission pairs in Georgia, USA, by using contact tracing information from COVID-19 cases reported to the Georgia Department of Public Health. The novel coronavirus disease 2019 (COVID-19) outbreak and Italy has caused 6088 cases and 41 deaths in Republic of Korea and 3144 cases and 107 deaths in Italy by 5 March 2020. Currently, the following variants; Alpha, Beta, Gamma, Delta and Omicron were originated from United Kingdom, South Africa, Brazil/Japan, and India, respectively. The classification of SARS-CoV-2 variants is based on the mutations in the structure-based inclusions. Transmission varied not only by time and place but also by persons' sex and race. Younger adults (20-50 years of age) were involved in most transmission events.
\end{abstract}

Keywords: Coronavirus disease 2019 (COVID-19); Transmission; Time and place; Sex and Race; SRS-CoV-2; Variants

\footnotetext{
${ }^{*}$ Corresponding author: Mohammed Farhan Qureshi

Prince Sultan Military Medical City, Division of Neonatology, Department of Pediatrics, Riyadh, Saudi Arabia. 


\section{Graphical abstract}

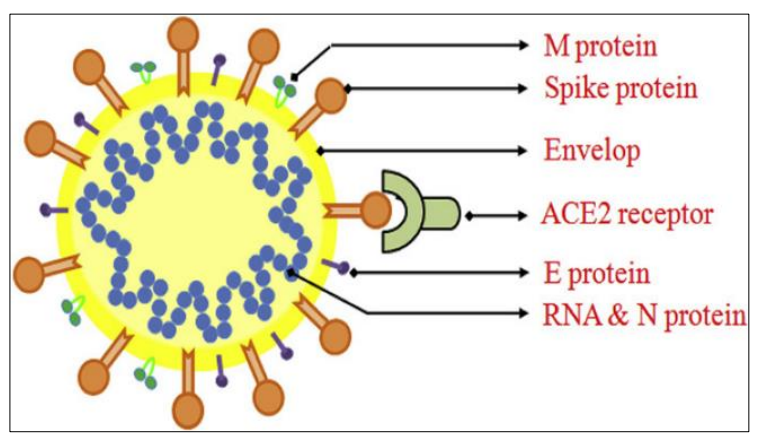

Figure Coronavirus (SARS-CoV-2)

\section{Introduction}

It is normal for viruses to change and evolve as they spread between people over time. When these changes become significantly different from the original virus, they are known as "variants." To identify variants, scientists map the genetic material of viruses (known as sequencing) and then look for differences between them to see if they have changed. Since the SARS-CoV-2 virus, the virus that causes COVID-19, has been spreading globally, variants have emerged and been identified in many countries around the world.

A new severe acute respiratory syndrome (SARS-CoV-2) associated with transmission (human to human) and extreme human sickness has been as of late announced from the city of Wuhan in China. Several genes of SARS-CoV-2, including $\mathrm{S}, \mathrm{N}$, and NSP12, present a high mutational range [1]. However, compared to other RNA viruses, coronaviruses present a low mutational frequency due to NSP14, which exhibits $3^{\prime}$ to $5^{\prime}$ Exonuclease activity that is critical for high viral replication fidelity [2]. It has been suggested that other factors can increase the number of mutations in SARS-CoV-2. For example, control measures have had a sizeable negative impact on the economy. Novel Coronavirus disease, COVID19 , was crowned as the second pandemic of the twenty-first century by the World Health Organization on March 11th, 2020. Most countries adopted incomplete or insufficient preventive/restrictive measures, with partial participation/adherence, resulting in ineffective control of the COVID-19 pandemic. Consequently, virus transmission and spread increased the probability of new mutations, leading to the emergence of variants with selective advantages [3]. Additionally, individuals with impaired immune competence suffer from prolonged SARS-CoV-2 infections, which increases the likelihood of new mutations [4]. There have also been many SARS-CoV-2 reinfections reported, raising immune pressure and selecting mutations that potentially can help escape immune defense [5]. Lastly, viral adaptation in susceptible animals and subsequent human infection can produce additional mutations in SARS-CoV-2 [5].

\section{Mutations in cellular inclusion of SARS-CoV-2 and creation of Different types Variants}

Mutations can occur in any region of the SARS-CoV-2 genome. Most of these mutations are silent, and hence do not modify the sequence of primary amino acids and the function of the translated proteins or viral infectivity. However, a single mutation, or a combination of mutations, can yield variants with survival and selective advantages and improved viral fitness. These mutational variants can present increased infectivity and/or transmissibility, human ACE-2 receptor binding affinity, viral replication, pathogenicity and reinfection risk. Moreover, depending on the location of the mutation, changes in antigenicity and host-, vaccine- or mAb-induced immune response evasion with alteration in crucial epitopes recognized by nAbs and/or decreased $\mathrm{T}$ cell immunity $[1,5,6]$.

While mutations in other genes can generate variants with increased viral replication, infectivity, and potential of the immune escape [7]. This review will focus on S gene mutations because the spike protein is the most enormously studied viral infection protein and the main protein target for vaccine development. For Example, recent studies have reported that R203K/G204R modifications in the N protein are linked with high viral infectivity, replication, and transmissibility in cellular and animal models [8]; it is observed that these alterations appear at a wide spread presence in the B.1.1.7 variant [9]. Corona virus NSP1 protein is important for suppressing interferon I signaling and increasing viral replication [10]. Notably, Lin et al. [11] observed that a deletion in NSP1 is linked to low plasma IFN- $\beta$ levels and viral load. The high prevalence of the P323L mutation in NSP12 has been implicated in viral replication [12]. 
Mutations in ORF8 have been suggested to increase viral transmission and immune evasion potential because its gene products participate in the RNA polymerase complex and are involved in controlling the host cells' major histocompatibility complex class I [13,14]. Pereira [14] observed a high prevalence of a premature stop codon at position 27 in ORF8 that occurs in the B.1.1.7 variant, positively contributing to its high transmission spread.

Emerging variants of SARS-CoV-2 can be considered a variant under investigation (VUI), a variant of interest (VOI), or a variant of concern (VOC). The WHO has recognized several VOIs, including B.1.427 and B.1.429 from the USA (California, WHO alert since July 6, 2021), B.1.525 from the UK and Nigeria, B.1.526 from the USA (New York), B.1.617.1 and B.1.617.3 from India, P2 from Brazil, and C.37 from Peru. Furthermore, the WHO has classified five variants as VOCs: B.1.1.7 from the UK (501Y. V1, VOC 202012/01, alpha variant), B.1.351 from South Africa (501Y. V2, VOC 202012/02, beta variant), P.1 from Brazil (501Y. V3, VOC 202101/02, gamma variant); B.1.617.2 from India (VOC 202104/02, delta), and B.1.1.529 from multiple countries (omicron variant). Notably, the B.1.617.2 variant was linked to the fast spread of SARS-CoV-2 in several countries [15].

The D614G mutant in the S gene, first identified in Europe in January 2020, was one of the first SARS-CoV-2 mutations to spread worldwide 960 [6]. This mutation is positioned between the S1 and S2 subunits and has been reported to increase in vitro viral infectivity [6]. affinity binding to the ACE-2 receptor and transmissibility [16], protease-induced S protein cleavage [17], replication, and viral loads [5]. Despite the apparently enhanced "viral fitness" [6] and its ability to neutralize the activity of antibodies induced by previous infections or vaccines [6]. The clinical outcomes or pathogenicity remain unchanged [16]. It has been proposed that the D614G mutation causes the ACE-2 receptor to assume "open conformation", increasing the binding affinity [18] and the virus's vulnerability to nAbs [19].

The B.1.1.298 variant was one of the first to contain the D614G mutation. This variant was linked with an outbreak on Denmark mink farms [20], resulting in 17 million Danish minks being culled as a preventive measure to stop virus evolution and spread [19]. It has been suggested that other modifications, including Y453F in the RBD of the S protein, P323L in NSP12 (a component of RdRp), and R203K and G204R in the N protein, also contributed to the improved viral fitness of the B.1.298 variant [21]. Notably, the D614G mutation has become more predominant, appearing in all recently identified variants.

The first VOC described (VOC 202012/01) was the B.1.1.7 lineage (20I/501Y. V1), identified in the UK. This variant is now present throughout the world. In December 2020, B.1.1.7 was responsible for one-quarter of the total COVID-19 cases worldwide and two-thirds of the cases in the UK [22]. Compared to the original virus, B.1.1.7 exhibits a 40-70\% increase in transmissibility [23]. The B.1.1.7 lineage has 23 mutations in the S, N, and ORF-8 genes, but the impact of each mutation on viral fitness and survival or vaccine efficacy is unknown [1,22].

The S protein of the B.1.1.7 lineage contains several amino acid mutations, including D614G and N501Y, and deletions [1]. The S RBD N501Y mutation increases the binding affinity to the ACE-2 receptor and transmissibility [1, 24].. Gu et al. [25] developed a mouse-adapted strain model (MASCp6) to evaluate the SARS-CoV-2 infectivity and virulence after intranasal inoculation and observed that the N501Y mutation favors interaction with ACE2 and promotes virus entry, consequently leading to enhanced virulence. Recent studies have suggested that the N501Y mutation has a low impact on clinical outcomes and pathogenicity [22] and the immune response generated by mAbs, vaccines, or previous infection $[1,26]$. However, Muik et al [26] evaluated more than 2.2 million people with SARS-CoV-2 positive tests and 17,452 related deaths in England and observed a 61\% higher risk of death in those infected with the B.1.1.7 variant than other pre-existing variants. Thus, the B.1.1.7 variant presents increased disease severity and transmissibility.

The B.1.351 lineage (20H/501Y, V2, VOC 202012/02) emerged in South Africa (Oct 2020), almost certainly favored by the high immune pressure, and spread to other African countries, Asia, Australia, and North and Central America [1]. By the end of 2020, B.1.351 was responsible for more than $90 \%$ of all COVID-19 cases in South Africa (28). This lineage has several structural and nonstructural mutations, including three critical mutations in the RBD of the S protein (K417N, E484K, and N501Y) that seem to play a crucial role in the improved "viral fitness" and survival adaptations compared to the other strains in some regions where it was prevalent [1]. The K417N mutation exacerbated immune escape from nabs and reduced vaccine effectivity against infection $[1,28]$, and the E484K modification is associated with increased binding to the ACE-2 receptor [1] and a decreased or even repeal response to Ab neutralization induced by previous infection, vaccination, or monoclonal Ab therapy [29]. Furthermore, five mutations in the NTD of the $S$ gene were proposed to contribute to improved viral microenvironment adaptations [30]. This lineage is also associated with increased spreading [19] and reinfection cases in subjects previously infected with the original SARS-CoV-2 [31].

In December 2020, the P.1 lineage (20J/501Y. V3, VOC 202101/02, also called P.1) accounted for 42\% of the total cases in Manaus, Brazil [3], and in February 2021, it was discovered in Japan in samples from individuals traveling from 
Manaus. The three main mutations are the same as in B.1.351: K417T, E484K, and N501Y [1]. This lineage has increased ACE-2 receptor binding affinity, transmissibility [1], infectivity in mice, and resistance to immune response and risk of reinfection [3].

The B.1.617 lineage contains three sub-lineages: B.1.617.1, B.617.2 (delta variant), and B.1.617.3. B1.617.2 exhibits higher transmissibility than the ancestral strain, and studies suggest a high risk of hospitalization compared to the original strain or the B.1.1.7 variant [32]. In a short review, Liu and Rocklov [32] reported a basic reproductive number of 5.08 for the delta variant versus 2.79 for the ancestral SARS-CoV-2 strain. Since no difference was observed in the median age and disease duration between patients infected with B.1.617.2 or non-B.1.617.2 strains [33], the elevated risk of hospitalization is probably due to the high transmissibility of the B.1.627.2 variant compared to other strains. Most fully vaccinated people are protected against the B.1.617 variants [34]. However, even after full vaccination, people can be infected by B.1.617.2 or other variants and transmit them to others, although with a lower risk of disease severity and reduced infection period than unvaccinated individuals [35]. Notable mutations in the B.1.617.2 variant include L452R, T478K and E484Q in the S RBD and P681R in the cleavage site between S1 and S2.

The L452R mutation appears to increase the interaction between RBD and the ACE-2 and infectivity [36]. Moreover, the T478K mutation, together with L452R, helps stabilize the RBD-ACE-2 complex and elevate the virus infectivity rate [37]. The E484Q mutation enhances binding affinity to ACE-2 and potentially reduces antibody binding affinity, an observation similar to the E484K mutation [36]. In addition, the P681R mutation, located at the cleavage site between S1 and S2, has been linked with augmented transmissibility and viral load [34]. In a preprint work, Liu et al. [38] observed that P681R modification leads to the increased furin cleavage site (S1-S2 junction), resulting in higher infectivity than the B.1.1.7 strain. The combination of mutations in the B.1.617.2 variant (delta variant) seems to impart the virus a selective advantage compared to the original virus and other variants, as evidenced by high transmissibility and infectivity, and potential immune evasion [34].

Recently, an emerging SARS-CoV-2 variant was initially identified in South Africa, but it has been simultaneously detected in several other countries. On November 26, 2021, the WHO classified this variant as a VOC (B.1.1.529, omicron variant) because of the alarming epidemiological situation in South Africa [39]. The B.1.1.529 variant contains several mutations present in other variants, such as N501Y (alpha), E484A E484K (beta and gamma), and T478K; P681H P681R (delta). Several of these changes observed in alpha, beta, and delta have been related to enhanced infectivity, transmissibility, and potential immune escape. However, it remains unclear whether or not the similarities with previous VOCs are related to the omicron variant's rapid spread. In total, the B.1.1.529 (omicron) variant has more than 50 mutations, with more than 30 in the $S$ gene alone [39]. In addition, Wang and Cheng [40] have identified potential mutations that can affect ACE2 and/or antibody binding. Omicron variant accumulates numerous mutations, including Q498R and S477N, which have been previously associated with elevated binding to ACE2 receptor, potentially enhancing viral infectivity to the host cells. Recently, it was observed a close connection between Omicron and the Alpha variants, suggesting that the omicron variant was circulating for a long period before its discovery [41]. Ongoing research is making attempts to make clear the role and effect of each mutation in the omicron variant. Currently, it appears as though the omicron variant does not increase disease severity or fatality (hospitalization and number of deaths), and there is no evidence of immune escape from approved vaccines.

In South Africa, the emergence of the omicron variant rapidly increased the number of daily cases from 273 cases/day on November 16 to more than 1,200 cases/day on November 25. Additionally, as of December 9, 2021, the omicron variant was confirmed in 63 countries in Africa, Europe, Australia, Asia and North, Central and South America [42].

\section{Efficacy and Antibody Neutralization Activity of Vaccines against SARS-CoV-2 Variants}

Most COVID-19 vaccines use the $S$ protein as the primary target, aiming to produce nAbs against the RBM regions, block the viral binding sites to the ACE-2 receptor in the host cells, and prevent infection [43]. Since the first generation of vaccines was developed based on the original SARS-CoV-2 without S protein amino acid mutations [6, 43]. medical scientists now face the challenge of determining if the efficacy of these vaccines against the new variants is maintained in its original state $[6,43]$. Presently, most approved COVID-19 vaccines protect against the described VOCs; however, constant surveillance and further studies about vaccine efficacies against the current VOCs and future SARS-CoV-2 variants are critical. It is also important to point out that most studies concerning nAbs activity were performed using SARS-CoV-2 pseudo-viruses, which may not reflect the virus's behavior in the real world. Furthermore, a reduction in $\mathrm{nAb}$ activity does not necessarily result in poor vaccine efficacy or effectiveness, as demonstrated by several recent studies. 
For example, the mRNA-based BNT $162 \mathrm{~b} 2$ vaccine (Pfizer/BioNTech) reached 95\% efficacy against the original SARCoV-2 infection [44]. Especially, using immune sera from vaccinated subjects, no difference [45] or mild to moderately decreased nAb activity (1.7 to 6.0-fold) against the B.1.1.7 pseudo-virus has been described. Thus, this variant probably does not increase immune escape or lessen the efficacy of vaccine (26). In contrast, the nAb activity provided by the BNT $162 \mathrm{~b} 2$ vaccine was significantly reduced (6.5 to 10.4-fold) or abrogated against the B.1.351 pseudo-virus [43]. Additionally, a reduction of 2.1 to 5.1 -fold and 1.4 to 3.0-fold in nAb activity was reported for the P.1 and B.1.617.2 variants, using the serum from vaccinated individuals [44, 45].

BNT 162b2 vaccine effectiveness was also evaluated in the Qatar population, when the B.1.1.7 and B.1.351 variants accounted for $50 \%$ and $44.5 \%$ of the total COVID-19 cases from February to March 2021 [46], A mass vaccination campaign was performed in the country, with 385,853 people receiving one dose and 265,410 receiving two doses of the vaccine by the end of March 2021 [46]. After 14 days or more after the second dose of the BNT $162 \mathrm{~b} 2$ vaccine, the effectiveness against B.1.1.7 variant infection was 89.5\%, and 75.0\% against B.1.351. Moreover, the vaccine's effectiveness against severe COVID-19 cases or death was $97.4 \%$ against both variants (46). In another real-world study performed in Qatar between December 2020 and September 2021, 950,232 people received at least one dose and 916,290 people two doses of the BNT162b2 vaccine (with an average of 21 days between doses) [47]. The authors observed similar results against infection (74.3\%) and severe/critical/fatal disease (92.7\%) caused by B.1.351 variant and low effectiveness against B.1.617.2 infection (51.9\%). Despite the reduced protection against infection, the vaccine was still highly effective against severe/critical/fatal disease (93.4\%) caused by the B.1.617.2 variant.

Another mRNA-based vaccine, the mRNA-1273 vaccine (Moderna), also reached a high global efficacy of $94 \%$ and induced nAb production (48). Against the B.1.1.7 pseudo-virus, no difference or a modest reduction in nAb activity was reported [48]. On the other hand, a pronounced reduction in nAb activity (6.4-fold) against the B.1.351 variant was observed [48]. Another study demonstrated that IgG antibody binding and neutralization activity are moderately impaired against the B.1.351 variant, but this vaccine is still efficient/effective against this variant [49]. Compared to B.1.351, the reduction in nAB activity was less pronounced in the P.1 and B.1.427/429 variants [48]. The mRNA-1273 vaccine also exhibited reduced $\mathrm{nAb}$ activity (2.1 to 3.3-fold) against B.1.617.2 compared to the D614G strain [50]. In the real-world study performed in Qatar described above [47], 564,468 people received at least one dose, and 509,322 received two doses of the mRNA-1273 vaccine (with an average of 28 days between doses). The authors reported $80.8 \%$ effectiveness against infection and $100 \%$ against severe, critical, or fatal disease caused by B.1.351 and $73.1 \%$ effectiveness against infection and 96.1\% against severe, critical, or fatal disease caused by B.1.167.2 variant.

The recombinant spike protein-based NVX-CoV2373 vaccine (Novavax) presents an efficacy of 95.6\% against the original SARS-CoV-2 strain [28]. Against the B.1.1.7 pseudo-virus, this vaccine has a low reduction in nAb activity [51], which is followed by a modest decrease in efficacy (85.6\%) [51]. In a South African phase 2a/b clinical trial, vaccine efficacy against mild to moderate disease was significantly reduced (49.4\%) in a population of 4,387 participants when the B.1.351 variant was predominant (92.7\%) [52]. In another study performed in South Africa, when more than $90 \%$ of the total COVID-19 cases were due to the B.1.351 variant (i.e., end of 2020 and the beginning of 2021), the vaccine reached an efficacy of 60\% (https://www.novavax.com/sites/default/files/2021-02/20210202-NYAS-NovavaxFinal.pdf). Currently, the B.1.617.2 variant is predominant in South Africa and worldwide, and data regarding effectiveness against this variant is crucial for understanding the real protection evoked by the Novavax vaccine.

The adenovirus vector-based ChAOx1-nCoV-19 vaccine (University of Oxford/AstraZeneca) was shown to have 66.7\% efficacy against SARS-CoV-2 infection [53]. Against the B.1.1.7 pseudo-virus vaccinee sera display reduced (9-fold and 2.5-fold) nAb activity without affecting vaccine efficacy (74.6\%) in 499 infected people with the variant [54]. Similar results were reported in another study comparing B.1.1.7 and non-B.1.1.7 lineages, with 70.4\% and 81.5\% efficacy against infection, respectively [55]. For the B.1.351 lineage, the ChAOx-1nCoV-19 vaccine elicits less potent nAb production against the B.1.351 pseudo-virus. B.1.351 is mainly characterized by the triple mutations in the RBD of the $\mathrm{S}$ protein and associated with reduced nAb titers (9-fold) and global efficacy against infection (10.4-20.4\%) and impaired efficacy (21.9\%) to prevent mild to moderate COVID-19 [54]. A small reduction in nAb activity was also reported for the P.1 (2.9-fold) and B.1.617.2 variants (5.0-fold) (55). Notably, the real-world effectiveness against B.1.617.2 infection (67\%) was similar to the wild-type (66.7\%) and B.1.1.7 (74.6\%) strains [34].

The Ad26.COV2. S or JNJ-78436735 vaccine (Janssen), another adenovirus vector-based vaccine, has an efficacy of $72 \%$ against B.1.1.7 infection. The vaccine's efficacy is reduced to $57 \%$ against the B.1.351 variant but is $89 \%$ effective at protecting against severe COVID-19 (https://www.jnj.com/johnson-johnson-announces-single-shot-janssen-covid-19vaccine-candidate-met-primary-endpoints-in-interim-analysis-of-its-phase-3-ensemble-trial). 
The vector-based Sputnik V vaccine or Gam-COVID-Vac (Gamaleya Institute) Moore and Offit, 2021) presents $91.6 \%$ efficacy against SARS-CoV-2 infection [56]. However, the efficacies against B.1.1.7, B.1.351, and P.1 were reduced to $81 \%, 59 \%$, and 52\%, respectively. The serum nAb activity was not significantly altered against B.1.1.7 but was decreased against the B.1.1.351 (3.1-fold), P.1 (2.8-fold), and B.1.617.2 (2.5-fold) variants [57].

The Sinopharm and Corona vaccines use inactivated virus-based technology. The CoronaVac vaccine was shown to be 83.5\% [58] and 65.9\% effective in studies conducted in Turkey and Chile, respectively [59]. It was also demonstrated that $\mathrm{nAb}$ activity was unaffected against B.1.429 but was decreased against the B.1.1.7, B.1.351, and P.1 variants by 2.0, 5.2, and 3.9-fold, respectively [40]. For the Sinopharm vaccine, nAb activity was reduced against the B.1.1.7 ( 2.0-fold) and B.1.351 (2.5 to 3.0-fold) variants [40].

More results related to the B.1.617.2 variant are necessary for all these vaccines to verify the real-world effectiveness against infection and severe or critical COVID-19 disease. Furthermore, a thorough analysis of this variant's potential immune response evasion in vaccinated individuals must be conducted. In relation to omicron variant, there are some preliminary results from few studies with limited sample size suggesting that the incidence of virus reinfection in South Africa can be associated with humoral (antibody-mediated) immune evasion and nAb activity in vaccinated or previously infected individuals [40].

Besides vaccines, convalescent sera have been used to evaluate the impact of variants on the nAb activity induced by the previous infection with the original SARS-CoV-2. Several studies observed a mild reduction ( 2.9 to 3.0 -fold) in $\mathrm{nAb}$ activity against the B.1.1.7 variant $(54,55)$. Partial (11 to $33 \mathrm{X}$ ). Variants containing the E484K mutation (e.g., B.1.351 and B.1.1.248 variants) were found to escape the immune response completely [54]. Additionally, convalescent sera of individuals infected with the original SARS-CoV-2 displayed impaired or nonexistent IgG antibody binding and neutralization activity against the B.1.351 variant (4 to 8-fold; 13.3-fold) [55], g eight months post-infection (2.1-fold) [55]. Indeed, it has been estimated that 41.1 to $48 \%$ of convalescent sera are incapable of neutralizing the B.1.351 pseudovirus [54]. Furthermore, a similar reduction in nAb activity was observed against the P.1 and B.1.1.7 variants (3.1 and 2.9-fold, respectively) [55].

\section{Variants of Concern and Potential Risk of New Pandemic Waves}

From December 2019 until December $16^{\text {th }}$ 2021, there have been more than 271,376,000 COVID-19 cases and 5,325,969 COVID-19-related deaths (1.96\% mortality rate) worldwide (World Health Organization, https://covid19.who.int). Moreover, approximately 3.4 million SARS-CoV-2 genome sequences have been submitted to the Global Initiative on Sharing All Influenza Data which has detected more than 4,100 mutations in the $S$ gene. About 1,200 of these mutations lead to amino acid substitutions, with 187 in the RBD of the S protein [1].

A monthly analysis of the VOC emergence was performed using the GISAID in several countries for one year (September 2020 to November 2021). The analysis of the epidemiological data of SARS-CoV-2 variants has several limitations, including (a) a limited number of genome sequencing data from a particular country; (b) samples from a particular group, city, or region that does not accurately represent the country; (c) the virus' behavior in a specific group, city or region; and (d) data release delay (data were extracted and analyzed on September 2, 2021, but new sequencing genomes are continuously submitted and updated, especially in the last few months). However, it provides a general overview of specific variants globally and highlights some important points.

For example, after the emergence of the B.1.1.7 variant in the UK (September 2020), it rapidly spread to several countries across all continents (169 countries on Sep 2, 2021). In 34 of the 67 countries analyzed, the B.1.1.7 variant became highly predominant, present at rates greater than $80 \%$; thus, demonstrating a clear selective advantage of this variant versus the original B.1 strain, which was the most prevalent strain at that moment in time. Additionally, in 12 of the 67 countries, this VOC was detected in 50.1 to $80 \%$ of the new monthly cases. In some countries, where other VOCs emerged before or even simultaneously, as in the case of B.1.351 in South Africa and Reunion and P.1 in Brazil, Chile, and French Guiana, the B.1.1.7 variant did not become predominant. This observation suggests that B.1.1.7 has no selective advantage over the B.1.351 and P.1 VOCs.

The B.1.351 variant emerged in South Africa in August 2020 and rapidly spread worldwide, reaching 111 countries as of September 2, 2021. Except for South Africa, Reunion, Angola, Philippines, Hong Kong, Bangladesh, and Qatar, the B.1.351 variant did not increase by more than $10 \%$ in most countries analyzed. A similar result was observed with the P.1 variant, which emerged in Brazil in December 2020. This variant disseminated at low rates $(<10 \%)$ in 78 countries (Sep 2, 2021) but had a high prevalence in Brazil, Chile, and French Guiana. 
The emergent B.1.617.2 variant appeared in India in October 2020. It has been dispersed throughout 147 countries (Sep 2,2021 ) and has a high predominance rate in most analyzed countries. For example, more than $80 \%$ of COVID-19 cases were B.1.617.2-induced in 52 of the 67 countries analyzed; 4 had between 50.1 to $80 \%$, and 11 had less than $50 \%$. Notably, there is an increasing trend in the countries with fewer B.1.617.2-related cases. Currently, this variant is the most prevalent VOC, displaying rapid transmission and spread and indicative of selective advantages against other VOCs such as B.1.1.7, B.1.351, and P.1. It will likely become predominant worldwide. Fortunately, B.1.617.2's high predominance has not increased the number of cases, hospitalizations or deaths, and the current vaccines effectively protect against all known VOCs.

\section{Conclusion}

The spike glycoprotein undergoes a constant process of genetic diversification that has led so far to the identification of a large variety of point mutations and deletions underlying the currently identified SARS-CoV-2 variants. Although experiments based on infection models are limited, there is clear evidence that spike mutations, particularly in the receptor binding domain, play a crucial role in modulating SARS-CoV-2's infectivity and antigenicity. Overall, this reinforces the need for ongoing molecular surveillance programs to guide the development and usage of vaccines and of therapeutics based on monoclonal antibodies and convalescent-phase sera. At the same time, the increasing circulation of variants with immune escape mutations supports the need to periodically update the formulation of the current vaccines and to test the efficacy of monoclonal antibodies in clinical use against newly arising variants in order to avoid potential loss of clinical efficacy.

\section{Compliance with ethical standards}

Disclosure of conflict of interest

There is no conflict of interest among any of the authors.

\section{References}

[1] Focosi D, Maggi F. Neutralising Antibody Escape of SARS-CoV-2 Spike Protein: Risk Assessment for AntibodyBased Covid-19 Therapeutics and Vaccines. Rev. Med. Virol. 2021; 31(6): e2231.

[2] Smith EC, Sexton NR, Denison MR. Thinking Outside the Triangle: Replication Fidelity of the Largest RNA Viruses. Annu. Rev. Virol. 2014; 1(1): 111-132.

[3] Chen J, Lu H. New Challenges to Fighting COVID-19: Virus Variants, Potential Vaccines, and Development of Antivirals. Biosci. Trends. 2021; 15(2): 126-128.

[4] Choi B, Choudhary MC, Regan J, Sparks JA, Padera RF, Qiu X, et al. Persistence and Evolution of SARS-CoV-2 in an Immunocompromised Host. N. Engl. J. Med. 2020; 383: 2291-2293.

[5] Abdel-Moneim AS, Abdelwhab EM, Memish ZA. Insights into SARS-CoV-2 Evolution, Potential Antivirals, and Vaccines. Virology. 2021; 558: 1-12.

[6] Dearlove B, Lewitus E, Bai H, Li Y, Reeves DB, Joyce MG, et al. A SARS-CoV-2 Vaccine Candidate Would Likely Match All Currently Circulating Variants. Proc. Natl. Acad. Sci. U. S. A. 2020; 117(38): 23652-23662.

[7] Khateeb J, Li Y, Zhang H. Emerging SARS-CoV-2 Variants of Concern and Potential Intervention Approaches. Crit. Care. 2021; 25(1): 244.

[8] Wu K, Werner AP, Koch M, Choi A, Narayanan E, Stewart-Jones GBE, et al. Serum Neutralizing Activity Elicited by mRNA-1273 Vaccine. N. Engl. J. Med. 2021; 384(15): 1468-1470.

[9] Collier DA, De Marco A, Ferreira IATM, Meng B, Datir RP, Walls AC, et al. Sensitivity of SARS-CoV-2 B.1.1.7 to mRNA Vaccine-Elicited Antibodies. Nature. 2021; 593(7857): 136-141.

[10] Xia H, Cao Z, Xie X, Zhang X, Chen JYC, Wang H, et al. Evasion of Type I Interferon by SARS-CoV-2. Cell Rep. 2020; 33: 108234.

[11] Lin J, Tang C, Wei H, Du B, Chen C, Wang M, et al. Genomic Monitoring of SARS-CoV-2 Uncovers an Nsp1 Deletion Variant That Modulates Type I Interferon Response. Cell Host Microbe. 2021; 29(3): 489-502.e8. 
[12] Koyama T, Platt D, Parida L. Variant Analysis of SARS-CoV-2 Genomes. Bull. World Health Organ. 2020; 98: 495504.

[13] Young BE, Fong SW, Chan YH, Mak TM, Ang LW, Anderson DE, et al. Effects of a Major Deletion in the SARS-CoV2 Genome on the Severity of Infection and the Inflammatory Response: An Observational Cohort Study. Lancet. 2020; 396: 603-611.

[14] Pereira F. SARS-CoV-2 Variants Combining Spike Mutations and the Absence of ORF8 may be More Transmissible and Require Close Monitoring. Biochem. Biophys. Res. Commun. 2021; 550: 8-14.

[15] Adam D. What Scientists Know About New, Fast-Spreading Coronavirus Variants? Nature. 2021; 594: 19-20.

[16] Volz E, Hill V, McCrone JT, Price A, Jorgensen D, O Toole A et al. Evaluating the Effects of SARS-CoV-2 Spike Mutation D614G on Transmissibility and Pathogenicity. Cell S0092-8674, 31537-31543. 2021.

[17] Gobeil SM, Janowska K, McDowell S, Mansouri K, Parks R, Manne K, et al. D614G Mutation Alters SARS-CoV-2 Spike Conformation and Enhances Protease Cleavage at the S1/S2 Junction. Cell Rep. 34, 108630. 2021.

[18] Yurkovetskiy L, Wang X, Pascal KE, Tomkins-Tinch C, Nyalile TP, Wang Y, et al. Structural and Functional Analysis of the D614G SARS-CoV-2 Spike Protein Variant. Cell. 2020; 183(3): 739-751.e8.

[19] Garcia-Beltran WF, Lam EC, Astudillo MG, Yang D, Miller TE, Feldman J, et al. COVID-19-Neutralizing Antibodies Predict Disease Severity and Survival. Cell. 2021(b); 184: 476-488.e11.

[20] Oude Munnink BB, Sikkema RS, Nieuwenhuijse DF, Molenaar RJ, Munger E, Molenkamp R, et al. Transmission of SARS-CoV-2 on Mink Farms be- Tween Humans and Mink and Back to Humans. Science. 2021; 371: 172-177.

[21] Plante JA, Liu Y, Liu J, Xia H, Johnson BA, Lokugamage KG, et al. Spike Mutation D614G Alters SARS-CoV-2 Fitness. Nature. 2020; 592(7852): 116-121.

[22] Conti P, Caraffa A, Gallenga CE, Kritas SK, Frydas I, Younes A, et al. The British Variant of the New Coronavirus19 (Sars-Cov-2) Should Not Create a Vaccine Problem. J. Biol. Regul. Homeost. Agents. 2021; 35(1): 1-4.

[23] Volz E, Mishra S, Chand M, Barrett JC, Johnson R, Geidelberg L, et al. Assessing Transmissibility of SARS-CoV-2 Lineage B.1.1.7 in England. Nature. 2021; 593(7858): 266-269.

[24] Starr TN, et al. Deep Mutational Scanning of SARS-CoV-2 Receptor Binding Domain Reveals Constraints on Folding and ACE2 Binding. Cell. 2020; 182: 1295-1310.e20.

[25] Gu H, Chen Q, Yang G, He L, Fan H, Deng YQ, et al. Adaptation of SARS-CoV-2 in BALB/c Mice for Testing Vaccine Efficacy. Science. 2020; 369: 1603-1607.

[26] Muik A, Wallisch AK, Sänger B, Swanson KA, Mühl J, Chen W, et al. Neutralization of SARS-CoV-2 Lineage B.1.1.7 Pseudovirus by BNT162b2 Vaccine-Elicited Human Sera. Science. 2021; 371(6534): 1152-1153.

[27] Davies NG, Jarvis CI, CMMID COVID-19 Working Group. Edmunds WJ, Jewell NP, Diaz-Ordaz K, et al. Increased Mortality in Community-Tested Cases of SARS-CoV-2 Lineage B.1.1.7. Nature. 2021; 593(7858): 2707-274.

[28] Callaway E, Mallapaty S. Novavax Offers First Evidence That COVID Vaccines Protect People Against Variants. Nature. 2021; 590(7844): 17.

[29] Liu Z, VanBlargan LA, Bloyet LM, Rothlauf PW, Chen RE, Stumpf S, et al. Identification of SARS-CoV-2 Spike Mutations That Attenuate Monoclonal and Serum Antibody Neutralization. Cell Host Microbe. 2021; 29(3): 477488.e4.

[30] Li Q, Nie J, Wu J, Zhang L, Ding R, Wang H, et al. SARS-CoV-2 501y.V2 Variants Lack Higher Infectivity Bu do Have Immune Escape. Cell. 2021; 184(9): 2362-2371.e9.

[31] Staub T, Arendt V, Lasso de la Vega EC, Braquet P, Michaux C, Kohnen M, et al. Case Series of Four Re-Infections With a SARS-CoV-2 B.1.351 Variant, Luxembourg, February 2021. Euro. Surveill. 2021; 26(18): 2100423.

[32] Liu Y, Rocklöv J. The Reproductive Number of the Delta Variant of SARS-CoV-2 Is Far Higher Compared to the Ancestral SARS-CoV-2 Virus. J. Travel. Med. 2021; 28(7): taab124.

[33] Mlcochova P, Kemp SA, Dhar MS, Papa G, Meng B, Ferreira IATM, et al. SARS-CoV-2 B.1.617.2 Delta Variant Replication and Immune Evasion. Nature. 2021; 599(7883): 114-119.

[34] Lopez Bernal J, Andrews N, Gower C, Gallagher E, Simmons R, Thelwall S, et al. Effectiveness of Covid-19 Vaccines Against the B.1.617.2 (Delta) Variant. N. Engl. J. Med. 2021; 385(7): 585-594.

[35] Ong SWX, Chiew CJ, Ang LW, Mak TM, Cui L, Toh MPHS et al. Clinical and Virological Features of SARS-CoV-2 Variants of Concern: A Retrospective Cohort Study Comparing B.1.1.7 (Alpha), B.1.315 (Beta) and B.1.617.2 (Delta). Clin. Infect. 2021 Aug 23; Ciab 721.

[36] Kirola L. Genetic Emergence of B.1.617.2 in COVID-19. New Microbes New Infect. 2021; 43: 100929. 
[37] Cherian S, Potdar V, Jadhav S, Yadav P, Gupta N, Das M, et al. SARS-CoV-2 Spike Mutations, L452R, T478K, E484Q and P681R, in the Second Wave of COVID-19 in Maharashtra, India. Microorganisms. 2021; 9(7): 1542.

[38] Liu J, Liu Y, Xia H, Zou J, Weaver SC, Swanson KA et al. BNT162b2-Elicited Neutralization of B.1.617 and Other SARS-CoV-2 Variants. Nature. 2021; 596(7871): 273-275.

[39] Callaway E. Heavily Mutated Omicron Variant Puts Scientists on Alert. Nature. 2021; 600(7887): 21.

[40] Wang L, Cheng G. Sequence Analysis of the Emerging SARS-CoV-2 Variant Omicron in South Africa. J. Med. Virol. 2021.

[41] Kandeel M, Mohamed MEM, Abd El-Lateef HM, Venugopala KN, El-Beltagi HS. Omicron Variant Genome Evolution and Phylogenetics. J. Med. Virol. 2021; $1-6$.

[42] Torjesen I. Covid-19: Omicron May Be More Transmissible Than Other Variants and Partly Resistant to Existing Vaccines, Scientists Fear. BMJ. 2021; 375: n2943.

[43] Chen RE, Zhang X, Case JB, Winkler ES, Liu Y, VanBlargan LA et al. Resistance of SARS-CoV-2 Variants to Neutralization by Monoclonal and Serum-Derived Polyclonal Antibodies. Nat. Med. 2021; 27(4): 717-726.

[44] Polack FP, Thomas SJ, Kitchin N, Absalon J, Gurtman A, Lockhart S, et al. Safety and Efficacy of the BNT162b2 mRNA Covid-19 Vaccine. N. Engl. J. Med. 2020; 383: 2603-2615.

[45] Kuzmina A, Khalaila Y, Voloshin O, Keren-Naus A, Boehm-Cohen L, Raviv Y, et al. SARS-CoV-2 Spike Variants Exhibit Differential Infectivity and Neutralization Resistance to Convalescent or Post-Vaccination Sera. Cell Host Microbe. 2021; 29(4): 522-528.e2.

[46] Abu-Raddad LJ, Chemaitelly H, Butt AA, National Study Group for COVID-19 Vaccination. Effectiveness of the BNT162b2 Covid-19 Vaccine against the B.1.1.7 and B.1.351 Variants. N. Engl. J. Med. 2021; 385(2): $187-189$.

[47] Tang P, Hasan MR, Chemaitelly H, Yassine HM, Benslimane FM, Al Khatib HA, et al. BNT162b2 and mRNA-1273 COVID-19 Vaccine Effectiveness Against the SARS-CoV-2 Delta Variant in Qatar. Nat. Med. 2021; 27(12): 21362143.

[48] Baden LR, El Sahly HM, Essink B, Kotloff K, Frey S, Novak R, et al. Efficacy and Safety of the mRNA-1273 SARSCoV-2 Vaccine. N. Engl. J. Med. 2021; 384: 403-416.

[49] Edara VV, Norwood C, Floyd K, Lai L, Davis-Gardner ME, Hudson WH, et al. Infection- and Vaccine-Induced Antibody Binding and Neutralization of the B.1.351 SARS-CoV-2 Variant. Cell Host Microbe. 2021(b); 29(4): 516521.

[50] Choi A, Koch M, Wu K, Dixon G, Oestreicher J, Legault H, et al. Serum Neutralizing Activity of mRNA-1273 Against SARS-CoV-2 Variants. J. Virol. 2021; 95 (23): e0131321.

[51] Shen X, Tang H, McDanal C, Wagh K, Fischer W, Theiler J, et al. SARS-CoV-2 Variant B.1.1.7 Is Susceptible to Neutralizing Antibodies Elicited by Ancestral Spike Vaccines. Cell Host Microbe. 2021; 29(4): 529-539.e3.

[52] Shinde V, Bhikha S, Hoosain Z, Archary M, Bhorat Q, Fairlie L, et al. Efficacy of NVX-CoV2373 Covid-19 Vaccine Against the B.1.351 Variant. N. Engl. J. Med. 2021; 384(20): 1899-1909.

[53] Voysey M, Clemens SAC, Madhi SA, Weckx LY, Folegatti PM, Aley PK, et al. Safety and Efficacy of the ChAdOx1 Ncov-19 Vaccine (AZD1222) Against SARS-CoV-2: An Interim Analysis of Four Randomised Controlled Trials in Brazil, South Africa, and the UK. Lancet. 2020; 397: 99-111.

[54] Zhou D, Dejnirattisai W, Supasa P, Liu C, Mentzer AJ, Ginn HM et al. Evidence of Escape of SARS-CoV-2 Variant B.1.351 From Natural and Vaccine-Induced Sera. Cell. 2021; 184(9): 2348-2361.e6.

[55] Dejnirattisai W, Zhou D, Supasa P, Liu C, Mentzer AJ, Ginn HM, et al. Antibody Evasion by the P.1 Strain of SARSCoV-2. Cell. 2021; 184(11): 2939-2954.e9.

[56] Logunov DY, Dolzhikova IV, Shcheblyakov DV, Tukhvatulin AI, Zubkova OV, Dzharullaeva AS, et al. Safety and Efficacy of a Rad26 and Rad5 Vector-Based Heterologous Prime-Boost COVID-19 Vaccine: An Interim Analysis of a Randomised Controlled Phase 3 Trial in Russia. Lancet. 2021; 397(10275): 671-681.

[57] Gushchin VA, Dolzhikova IV, Shchetinin AM, Odintsova AS, Siniavin AE, Nikiforova MA, et al. Neutralizing Activity of Sera from Sputnik V-Vaccinated People against Variants of Concern (VOC: B.1.1.7, B.1.351, P.1, B.1.617.2, B.1.617.3) and Moscow Endemic SARS-CoV-2 Variants. Vaccines. 2021; 9: 779.

[58] Tanriover MD, Doğanay HL, Akova M, Güner HR, Azap A, Akhan S, et al. Efficacy and Safety of an Inactivated Whole-Virion SARS-CoV-2 Vaccine (CoronaVac): Interim Results of a Double-Blind, Randomised, PlaceboControlled, and Phase 3 Trial in Turkey. Lancet. 2021; 398(10296): 213-222.

[59] Jara A, Undurraga EA, González C, Paredes F, Fontecilla T, Jara G, et al. Effectiveness of an Inactivated SARS-CoV2 Vaccine in Chile. N. Engl. J. Med. 2021; 385(10): 875-884. 